\title{
A NEW MEASURE OF ROTATABILITY OF RESPONSE SURFACE DESIGNS
}

\author{
Anant M. KshirsagaR ${ }^{1}$ and Joanna Chung-in Cheng ${ }^{1}$ \\ University of Michigan
}

\begin{abstract}
Summary
This paper proposes a new measure of rotatability of a response surface design. It is based on a comparison between the design moments of a given design and the design moments of a rotatable design closest to the given design in the least squares sense. This measure is easier to calculate than Khuri's measure. An alternative easier non-geometrical way of deriving Khuri's measure is also presented. Both measures are calculated to illustrate some designs considered by Khuri.
\end{abstract}

Key words: Rotatability; response surface designs; design moments.

\section{Introduction}

Rotatability as defined by Box \& Hunter (1957) is an important property of response surface designs. It ensures equal precision of estimated responses at equal distances from the centroid of the design. Box \& Hunter derived certain conditions on the moments of a design matrix that must be satisfied for rotatability. Khuri (1988) and Draper \& Gutterman (1988) have suggested measures of departure from rotatability. Khuri uses a geometrical approach, projecting a vector associated with the design matrix on the cone of rotatability, after expressing the vector in a canonical form.

In this paper we suggest an alternative measure of the rotatability of a design. This measure is based on the design moments and is easier to calculate. We given an explicit formula for this measure and compare it with Khuri's measure. Incidentally, we derive Khuri's measure by an alternative analytical method that does not require geometrical concepts.

\section{Moments of a Design}

Consider a response surface model of degree $d$ in the levels $x_{1}, \ldots, x_{k}$ of $k$ factors and $N$ experimental runs. The model for the response variable $y$ is then

$$
\mathrm{E}(y)=\mathrm{x}^{[d]^{\prime}} \boldsymbol{\beta},
$$

Received October 1995; accepted February 1996.

${ }^{1}$ Dept of Biostatistics, School of Public Health, University of Michigan, 1420 Washington Heights, Ann Arbor, Michigan 48109-2029, USA. 
where $\mathbf{x}^{[d]}$ is the 'derived power vector of degree $d$ ' or Schlaffian associated with the vector

$$
\mathbf{x}^{\prime}=\left[1, x_{1}, \ldots, x_{k}\right]
$$

as defined by Box \& Hunter (1957) (see also Myers, 1971). It is in fact a vector such that

$$
\mathbf{x}^{[d]} \mathbf{x}^{[d]}=\left(\mathbf{x}^{\prime} \mathbf{x}\right)^{d} \text {. }
$$

For example, $x^{[2]^{\prime}}=\left[1, \sqrt{2} x_{1}, \ldots, \sqrt{2} x_{k}, x_{1}^{2}, \ldots, \sqrt{2} x_{1} x_{2}, \ldots\right]$ and is obtained from the terms in the expansion of $\left(\mathrm{x}^{\prime} \mathbf{x}\right)^{d}$. Let $x_{i u}$ be the level of the $i$ th factor in the $u$ th run and let $X$ be a matrix with $N$ rows, the $u$ th row of which equals $\mathbf{x}_{u}^{[d]^{\prime}}$. The moment of order $\delta=\delta_{1}+\cdots+\delta_{k}$ of this design is then

$$
M\left(\delta_{1}, \ldots, \delta_{k}\right)=\sum_{u=1}^{N} x_{1 u}^{\delta_{1}} \ldots x_{k u}^{\delta_{k}}
$$

If $\delta$ is even, this is called an even moment. Box \& Hunter showed that the design moments of such a design are obtained from the generating function

$$
\Phi(t)=t^{\left[d d^{\prime}\right.} X^{\prime} X t^{[d]}=\sum_{u=1}^{N}\left(1+\sum_{i=1}^{k} t_{i} x_{u i}\right)^{2 d}
$$

where $\mathbf{t}^{\prime}=\left[1, t_{1}, \ldots, t_{k}\right]$. The coefficient of $t_{1}^{\delta_{1}} \ldots t_{k}^{\delta_{k}}$ in $\Phi(\mathbf{t})$ is

$$
a\left(\delta_{1}, \ldots, \delta_{k}\right) M\left(\delta_{1}, \ldots, \delta_{k}\right) \quad(\delta \leq 2 d)
$$

where

$$
a\left(\delta_{1}, \ldots, \delta_{k}\right)=\frac{(2 d) !}{(2 d-\delta) ! \prod_{i=1}^{k}\left(\delta_{i} !\right)} .
$$

For a rotatable design Box \& Hunter showed that $\Phi(t)$ is of the form

$$
\Phi_{R}(t)=\sum_{j=0}^{d} a_{2 j}\left(\sum_{i=1}^{k} t_{i}^{2}\right)^{j}
$$

for some $a_{2 j} \mathrm{~s}$. Therefore the moments $M_{R}\left(\delta_{1}, \ldots, \delta_{k}\right)$ of a rotatable design are of the form

$$
M_{R}\left(\delta_{1}, \ldots, \delta_{k}\right)=\theta_{\delta} C\left(\delta_{1}, \ldots, \delta_{k}\right) I\left(\delta_{1}, \ldots, \delta_{k}\right),
$$

where

$$
\theta_{\delta}=\frac{a_{\delta} 2^{\delta / 2}(\delta / 2) !(2 d-\delta) !}{(2 d) !}
$$




$$
\begin{aligned}
C\left(\delta_{1}, \ldots, \delta_{k}\right) & =\frac{\prod_{i} \delta_{i} !}{2^{\delta / 2} \prod_{i}\left(\delta_{i} / 2\right) !}, \\
I\left(\delta_{1}, \ldots, \delta_{k}\right) & = \begin{cases}1 & \text { if every } \delta_{i} \text { is even, } \\
0 & \text { otherwise. }\end{cases}
\end{aligned}
$$

As in Khuri (1988) we assume that $\sum_{u} x_{i u}=0, \sum x_{i u}^{2}=1$ for all $i$; otherwise we define

$$
x_{i u}=\frac{x_{i u}^{*}-\bar{x}_{i}^{*}}{\left[\sum_{u=1}^{N}\left(x_{i u}^{*}-\bar{x}_{i}^{*}\right)^{2}\right]^{1 / 2}}
$$

for the original levels $x_{i u}^{*}$ and work with these transformed values. This, as Khuri (1988) explains, is necessary to have our measures of rotatability invariant with respect to origin and scale. As a result, in addition to the obvious property $M(0, \ldots, 0)=N$, our design $D$ has

$$
M(0, \ldots, 0,1, \ldots, 0)=0, \quad M(0, \ldots, 0,2,0, \ldots, 0)=1
$$

where the 1 and 2 in the last two moments are in the $i$ th places $(i=1, \ldots, k)$.

\section{: 3. A New Measure of Rotatability}

To define this measure of rotatability for the design $D$, we consider a rotatable design $D_{R}$, for which

$$
M_{R}(0, \ldots, 0,1, \ldots, 0)=0, \quad M_{R}(0, \ldots, 0,2,0, \ldots, 0)=1
$$

(like those moments of $D$ ) and the arbitrary $\theta_{\delta}\left(\right.$ or $a_{\delta}$ ) in its other moments are such that the sum of squares of the differences in the coefficients of $t_{1}^{\delta_{1}} \ldots t_{k}^{\delta_{k}}$ in $\Phi(t)$ and $\Phi_{R}(t)$ is minimum. The design $D_{R}$ is then closest to the given design $D$ in a least squares sense. The quantity

$$
\begin{aligned}
\Psi & =\sum a^{2}\left(\delta_{1}, \ldots, \delta_{k}\right)\left[M\left(\delta_{1}, \ldots, \delta_{k}\right)-M_{R}\left(\delta_{1}, \ldots, \delta_{k}\right)\right]^{2} \\
& =\sum a^{2}\left(\delta_{1}, \ldots, \delta_{k}\right)\left[M\left(\delta_{1}, \ldots, \delta_{k}\right)-\theta_{\delta} C\left(\delta_{1}, \ldots, \delta_{k}\right) I\left(\delta_{1}, \ldots, \delta_{k}\right)\right]^{2},
\end{aligned}
$$

where the summation extends over all $\left(\delta_{1}, \ldots, \delta_{k}\right)$ and is thus minimised with respect to $\theta_{\delta}$. Separating the odd and even order moments in $\Psi$, excluding all $\left(\delta_{1}, \ldots, \delta_{k}\right)$ such that $\delta=0$ or 1 (as $M=M_{R}$ for these values) and observing that $M_{R}=0$ if $\delta$ is odd, we can easily see that the value of $\theta_{\delta}$ that minimises $\Psi$ and gives the rotatable design $D_{\tau}$ that is closest to $D$, is

$$
\hat{\theta}_{\delta}=\frac{\sum^{\prime \prime} M\left(\delta_{1}, \ldots, \delta_{k}\right) C\left(\delta_{1}, \ldots, \delta_{k}\right) /\left(\prod \delta_{i} !\right)^{2}}{\sum^{\prime \prime} C^{2}\left(\delta_{1}, \ldots, \delta_{k}\right) /\left(\Pi \delta_{i} !\right)^{2}},
$$


where $\sum^{\prime \prime}$ denotes summation over all $\left(\delta_{1}, \ldots, \delta_{k}\right)$ such that each $\delta_{i}$ is even or zero and $\sum \delta_{i}=\delta$. The minimum value of $\Psi$, using this optimum $\hat{\theta}_{\delta}$ is easily seen to be

$$
\Psi_{\min }=\sum a^{2}\left(\delta_{1}, \ldots, \delta_{k}\right) M^{2}\left(\delta_{1}, \ldots, \delta_{k}\right)-\sum_{\delta=4}^{2 d} \sum^{\prime \prime} \hat{\theta}_{\delta}^{2}\left(\delta_{1}, \ldots, \delta_{k}\right) C^{2}\left(\delta_{1}, \ldots, \delta_{k}\right),
$$

where the first summation $\sum$ is over all $\left(\delta_{1}, \ldots, \delta_{k}\right)$ except when $\delta=0$ and $\delta=1$. A measure of rotatability then should compare this $\Psi_{\min }$ with the sum of squares of the coefficients of $t_{1}^{\delta_{1}} \ldots t_{k}^{\delta_{k}}$ in $\Phi(t)$ of the given design. We therefore propose

$$
R=\frac{\sum_{\delta=4}^{2 d} \sum^{\prime \prime} \hat{\theta}_{\delta}^{2} a^{2}\left(\delta_{1}, \ldots, \delta_{k}\right) C^{2}\left(\delta_{1}, \ldots, \delta_{k}\right)}{\sum^{\prime} a^{2}\left(\delta_{1}, \ldots, \delta_{k}\right) M^{2}\left(\delta_{1}, \ldots, \delta_{k}\right)} \times 100 \% .
$$

The summation in the denominator is over all $\left(\delta_{1}, \ldots, \delta_{k}\right)$ except when $\delta=0$, $\delta=1$ or when only one $\delta_{i}=2$, the rest being all equal to 0 .

\section{Khuri's Measure of Rotatability}

The matrix $X$ we defined in Section 1 has $\mathbf{x}_{u}^{[d]^{\prime}}$ as its $u$ th row. Khuri defines his matrix $X$ in a slightly different way. It has, in the $u$ th row, the elements $1, x_{1 u}, x_{2 u}, \ldots, x_{k u}, x_{1 u}^{2}, x_{2 u}^{2}, \ldots, x_{1 u} x_{2 u}, \ldots$ etc. Each element is a product of powers of $x_{i u}(i=1, \ldots, k)$ such that the degree is $d$ or less. The entries in our matrix $X$ are algebraically very like those of Khuri, apart from the numerical coefficients, which in our case are not all unity. For Khuri's matrix, $X^{\prime} X$ has the elements $M\left(\delta_{1}, \ldots, \delta_{k}\right)$ and they occur different numbers of times in $X^{\prime} X$. Let $f\left(\delta_{1}, \ldots, \delta_{k}\right)$ be the number of times $M\left(\delta_{1}, \ldots, \delta_{k}\right)$ occurs on the diagonal of $X^{\prime} X$ or above it. Khuri then considers the sum of squares $\Psi_{k}$ of differences of the elements of his $X^{\prime} X$ (on the diagonal and above it) with the corresponding elements of the $X^{\prime} X$ matrix of the closest rotatable design $D_{R}$, excluding the moments $M(0, \ldots, 0), M(0, \ldots, 1,0 \ldots, 0)$ (1 in the $i$ th place; $i=1, \ldots, k)$, $M(0, \ldots, 0,2,0, \ldots, 0),(2$ in the $i$ th place; $i=1, \ldots, k)$. He uses a vec notation. But all this amounts to minimising (with respect to $\theta_{\delta}$ )

$$
\Psi_{k}=\sum f\left(\delta_{1}, \ldots, \delta_{k}\right)\left[M\left(\delta_{1}, \ldots, \delta_{k}\right)-C\left(\delta_{1}, \ldots, \delta_{k}\right) \theta_{\delta} I\left(\delta_{1}, \ldots, \delta_{k}\right)\right]^{2}
$$

where the summation is over all $\left(\delta_{1}, \ldots, \delta_{k}\right)$ except those corresponding to moments of order 0,1 and pure moments of order 2 . This gives the closest rotatable design $D_{R}$. Khuri's measure $K$ has an expression very similar to our $R$, except that $a\left(\delta_{1}, \ldots, \delta_{k}\right)$ in (3.4) is replaced by $f\left(\delta_{1}, \ldots, \delta_{k}\right)$. Both these measures $R$ and $K$ are, in a sense, weighted averages of squared differences of the moments of $D$ and those of the closest $D_{R}$. Only the weights are different. The measure $R$ has the advantage that $a\left(\delta_{1}, \ldots, \delta_{k}\right)$ has an explicit formula but for Khuri's $K, f\left(\delta_{1}, \ldots, \delta_{k}\right)$ must be counted by actually writing down $X^{\prime} X$. 
TABLE 1

Moments of $D$ and $D_{R}$; frequencies of $f$ and coefficients a

\begin{tabular}{lccrl}
\hline Moment & $f\left(\delta_{1}, \delta_{2}\right)$ & $\delta$ & $a\left(\delta_{1}, \delta_{2}\right)$ & $M_{R}\left(\delta_{1}, \delta_{2}\right)=\theta_{\delta} C\left(\delta_{1}, \delta_{2}\right) I$ \\
\hline$M(0,0)=n$ & 1 & 0 & 1 & $M_{R}(0,0)=n$ \\
$M(1,0)=M(0,1)=0$ & 1 & 1 & 4 & $M_{R}(1,0)=M_{R}(0,1)=0$ \\
$M(1,1)$ & 2 & 2 & 12 & $M_{R}(1,1)=0$ \\
$M(2,0)=M(0,2)=1$ & 2 & 2 & 6 & $M_{R}(2,0)=M_{R}(0,2)=1$ \\
$M(2,1), M(1,2)$ & 2 & 3 & 12 & $M(2,1)=M(1,2)=0$ \\
$M(2,2)$ & 2 & 4 & 6 & $\theta_{4}$ \\
$M(3,0), M(0,3)$ & 1 & 3 & 4 & $M_{R}(3,0)=M_{R}(0,3)=0$ \\
$M(3,1), M(1,3)$ & 1 & 4 & 4 & $M_{R}(3,1)=M_{R}(1,3)=0$ \\
$M(4,0), M(0,4)$ & 1 & 4 & 1 & $M_{R}(4,0)=M_{R}(0,4)=3 \theta_{4}$ \\
\hline
\end{tabular}

\section{Rotatability of a Second Order Design, with Two Factors}

For $d=2$ and $k=2$, Khuri's $X^{\prime} X$ matrix (when $\sum x_{i}=0, \sum x_{i}^{2}=1$ ) is

$$
\left[\begin{array}{cccccc}
n & 0 & 0 & 1 & 1 & M(1,1) \\
& 1 & M(1,1) & M(2,0) & M(1,2) & M(2,1) \\
& 1 & M(2,1) & M(0,3) & M(1,2) \\
& & M(4,0) & M(2,2) & M(3,1) \\
& & & M(0,4) & M(1,3) \\
& & & & & M(2,2)
\end{array}\right]
$$

Table 1 shows the frequencies $f\left(\delta_{1}, \delta_{2}\right)$ of $M\left(\delta_{1}, \delta_{2}\right)$ in the above matrix and the coefficients $a\left(\delta_{1}, \delta_{2}\right)$ as defined in (2.1), and the corresponding values $M_{R}\left(\delta_{1}, \delta_{2}\right)$ of a rotatable design, as given by (2.2).

Khuri's $\Psi_{k}$ then becomes

$$
\begin{aligned}
\Psi_{k}=\sum & f\left(\delta_{1}, \delta_{2}\right)\left[M\left(\delta_{1}, \delta_{2}\right)-M_{R}\left(\delta_{1}, \delta_{2}\right)\right]^{2} \\
= & 2 M^{2}(1,1)+2 M^{2}(2,1)+2 M^{2}(1,2)+M^{2}(3,0)+M^{2}(0,3) \\
& +M^{2}(3,1)+M^{2}(1,3)+2\left[M(2,2)-\theta_{4}\right]^{2} \\
& +\left[M(4,0)-3 \theta_{4}\right]^{2}+\left[M(0,4)-3 \theta_{4}\right]^{2} .
\end{aligned}
$$

Minimising $\Psi_{k}$ with respect to $\theta_{4}$, we find that the closest $D_{R}$ has

$$
\hat{\theta}_{4}=\frac{1}{20}[2 M(2,2)+3 M(4,0)+3 M(0,4)] \text {. }
$$

Hence,

$$
K=\frac{100 \% \times 20 \times \hat{\theta}_{4}^{2}}{\sum_{\delta=2}^{4} f\left(\delta_{1}, \delta_{2}\right) M^{2}\left(\delta_{1}, \delta_{2}\right)},
$$


TABLE 2

Roquemore designs

\begin{tabular}{cccc}
\hline Design & 310 & $311 \mathrm{~A}$ & $311 \mathrm{~B}$ \\
\hline$K$ & $94.89 \%$ & $99.40 \%$ & $98.99 \%$ \\
$R$ & $97.16 \%$ & $99.82 \%$ & $98.46 \%$ \\
\hline
\end{tabular}

where the summation $\Sigma^{\prime}$ excludes $M(0,2)$ and $M(2,0)$. Therefore,

$$
\begin{array}{r}
K=\frac{100 \% \times[2 M(2,2)+3 M(4,0)+3 M(0,4)]^{2}}{20\left[2 M^{2}(1,1)+2 M^{2}(1,2)+2 M^{2}(2,1)+2 M^{2}(2,2)+M^{2}(3,0)\right.} \\
\left.+M^{2}(0,3)+M^{2}(3,1)+M^{2}(1,3)+M^{2}(4,0)+M^{2}(0,4)\right]
\end{array}
$$

The optimum value of $\theta_{4}$ in the measure $R$ we propose is obtained by minimising

$$
\begin{aligned}
\Psi=14 & {\left[M^{2}(1,1)+M^{2}(2,1)+M^{2}(1,2)\right]+36\left[M(2,2)-\theta_{4}\right]^{2} } \\
& +16\left[M^{2}(3,0)+M^{2}(0,3)\right]+16\left[M^{2}(3,1)+M^{2}(1,3)\right] \\
& +\left[M(4,0)-3 \theta_{4}\right]^{2}+\left[M(0,4)-3 \theta_{4}\right]^{2}
\end{aligned}
$$

with respect to $\theta_{4}$, yielding

$$
\tilde{\theta}_{4}=\frac{1}{18}[M(4,0)+M(0,4)+12 M(2,2)] .
$$

Substituting this in $R$ we find,

$$
R=\frac{100 \% \times \frac{1}{6}[M(4,0)+M(0,4)+12 M(2,2)]^{2}}{\left(144\left[M^{2}(1,1)+M^{2}(2,1)+M^{2}(1,2)\right)+36\left[M^{2}(2,2)+16\left[M^{2}(3,0)\right.\right.\right.} .
$$

We use these expressions to find the measure of rotatability of the $3^{2}$ factorial design considered by Khuri that has $n=9, M(1,1)=M(1,2)=M(2,1)=$ $M(3,0)=M(0,3)=M(1,3)=M(3,1)=0, M(2,2)=1 / 9$ and $M(4,0)=$ $M(0,4)=1 / 6$. From (5.1) and (5.2), for this design, the rotatability index is

$$
K=93.08 \%, \quad R=92.60 \% \text {. }
$$

Khuri has calculated $K$ for Roquemore (1976) designs with $k=3$ and $d=2$. We give $K$ and $R$ for these three designs in Table 2 .

Finally we consider Scheffé's (1958) mixture design with $k=3$ factors and the model

$$
\mathrm{E}(y)=\beta_{1} x_{1}+\beta_{2} x_{2}+\beta_{3} x_{3}+\beta_{12} x_{1} x_{2}+\beta_{13} x_{1} x_{3}+\beta_{23} x_{2} x_{3}
$$


For the design, the vertex point, i.e. a point of the type

$$
x_{i}=1, \quad x_{j}=0 \quad(i \neq j, i, j=1,2,3),
$$

is repeated $r_{a}$ times; the mid-point, i.e. a point of the type

$$
x_{i}=x_{j}=\frac{1}{2}, \quad x_{k}=0 \quad(i \neq j \neq k),
$$

is repeated $r_{b}$ times. Cheng (1991) showed that for this design,

$$
R=\frac{3 b^{2}}{b^{2}+68 a^{2}+1}, \quad \text { while } \quad K=\frac{4 b^{2}}{4 b^{2}+24 a^{2}+1},
$$

where

$$
a=\frac{1}{9 c^{3 / 2}}\left(-r_{a}+\frac{1}{8} r_{b}\right), \quad b=\frac{1}{9 c^{2}}\left(r_{a}+\frac{1}{16} r_{b}\right), \quad c=\frac{2}{3} r_{a}+\frac{1}{6} r_{b} .
$$

\section{Conclusions}

Note that both the measures of $K$ and $R$ are weighted averages of the squared differences between moments of a given design and those of the closest rotatable design, but the advantage of $R$ is that the weights have an explicit formula, while for $k$ one needs to write the whole $X^{\prime} X$ matrix and count the frequencies $f\left(\delta_{1}, \ldots, \delta_{k}\right)$ of the different moments. Our measure $R$ should prove useful in algorithms for the automatic construction of near-optimal designs where approximate rotatability is a desirable additional property.

\section{References}

BOX, G.E.P. \& HUNTER, J.S. (1957). Multifactor experimental designs for exploring response surfaces. Ann. Math. Statist. 28, 195-241.

CHENG, JOANNA CHUNG-IN. (1991). Some Aspects of Mixture and Response Surface Designs. PhD dissertation, Dept of Biostatistics, University of Michigan, Ann Arbor.

DRAPER, N.R. \& GUTTERMAN, I. (1988). An index of rotatability. Technometrics 30, 105-111.

KHURI, A.I. (1988). A measure of rotatability for response-surface designs. Technometrics 30 , 95-104.

MYERS, R.H. (1971). Response Surface Methodology. Boston: Allyn and Bacon.

ROQUEMORE, K.G. (1976). Hybrid designs for quadratic response surfaces. Technometrics $18,419-423$.

SCHEFFE, H. (1958). Experiments with mixtures. J. Roy. Statist. Soc. Ser. B 20, 344-360. 